CLINICAL STUDY

\title{
The impact of low triiodothyronine levels on mortality is mediated by malnutrition and cardiac dysfunction in incident hemodialysis patients
}

\author{
Hyang Mo Koo ${ }^{1}$, Chan Ho Kim ${ }^{1}$, Fa Mee Doh ${ }^{1}$, Mi Jung Lee ${ }^{1}$, Eun Jin Kim ${ }^{1}$, Jae Hyun Han ${ }^{1}$, Ji Suk Han ${ }^{1}$, \\ Hyung Jung $\mathrm{Oh}^{1}$, Seung Hyeok Han ${ }^{1}$, Tae-Hyun Yoo $^{1}$ and Shin-Wook Kang ${ }^{1,2}$ \\ ${ }^{1}$ Department of Internal Medicine, College of Medicine and ${ }^{2}$ Severance Biomedical Science Institute, Brain Korea 21, Yonsei University, Seoul, Korea \\ (Correspondence should be addressed to $S$-W Kang who is now at Department of Internal Medicine, College of Medicine, Severance Biomedical Science \\ Institute, Brain Korea 21, Yonsei University, 134 Shinchon-Dong, Seodaemoon-Gu, Seoul 120-752, Korea; Email: kswkidney@yuhs.ac)
}

\begin{abstract}
Objective: Little is known about the impact of low triiodothyronine $\left(\mathrm{T}_{3}\right)$ levels on mortality in end-stage renal disease (ESRD) patients starting hemodialysis (HD) and whether this impact is mediated by malnutrition, inflammation, or cardiac dysfunction.

Design and methods: A prospective cohort of 471 incident HD patients from 36 dialysis centers within the Clinical Research Center for ESRD in Korea was selected for this study. Based on the median value of $\mathrm{T}_{3}$, patients were divided into 'higher' and 'lower' groups, and all-cause and cardiovascular $(\mathrm{CV})$ mortality rates were compared. In addition, associations between $\mathrm{T}_{3}$ levels and various nutritional, inflammatory, and echocardiographic parameters were determined.

Results: Compared with those in the 'higher' $\mathrm{T}_{3}$ group, albumin, cholesterol, and triglyceride levels, lean body mass estimated by creatinine kinetics (LBM-Cr), and normalized protein catabolic rate (nPCR) were significantly lower in patients with 'lower' $\mathrm{T}_{3}$ levels. The 'lower' $\mathrm{T}_{3}$ group also had a higher left ventricular mass index (LVMI) and a lower ejection fraction (EF). Furthermore, correlation analysis revealed significant associations between $\mathrm{T}_{3}$ levels and nutritional and echocardiographic parameters. All-cause and CV mortality rates were significantly higher in patients with 'lower' $\mathrm{T}_{3}$ levels than in the 'higher' $\mathrm{T}_{3}$ group (113.4 vs 18.2 events per 1000 patient-years, $P<0.001$, and 49.8 vs 9.1 events per 1000 patient-years, $P=0.001$, respectively). The Kaplan-Meier analysis also showed significantly worse cumulative survival rates in the 'lower' $\mathrm{T}_{3}$ group $(P<0.001)$. In the Cox regression analysis, low $\mathrm{T}_{3}$ was an independent predictor of all-cause mortality even after adjusting for traditional risk factors (hazard ratio $=3.76, P=0.021$ ). However, the significant impact of low $\mathrm{T}_{3}$ on all-cause mortality disappeared when LBM-Cr, nPCR, LVMI, or EF were incorporated into the models.

Conclusion: Low $\mathrm{T}_{3}$ has an impact on all-cause mortality in incident HD patients, partly via malnutrition and cardiac dysfunction.
\end{abstract}

European Journal of Endocrinology 169 409-419

\section{Introduction}

Thyroid hormone has numerous effects on the kidney, playing an important role in renal development and growth as well as in sodium and water homeostasis. Renal plasma flow (RPF) and glomerular filtration rate (GFR) are also influenced by thyroid hormone. As a result, reductions in RPF and GFR, an increase in serum creatinine concentrations, and hyponatremia are frequently observed in primary hypothyroidism patients, and these renal derangements can be nearly normalized by thyroid hormone replacement therapy $(1,2,3)$.

Conversely, the kidney is involved in the metabolism and excretion of thyroid hormone; thus, renal failure can lead to significant changes in thyroid function. Goiter, thyroid nodules, reduced total and free
3,5,3'-triiodo-L-thyronine ( $\left.\mathrm{f}_{3}\right)$ and thyroxine $\left(\mathrm{T}_{4}\right)$ levels, and subclinical hypothyroidism are not uncommon in patients with chronic kidney disease (CKD). Among these abnormalities, reduced serum $\mathrm{T}_{3}$ concentration (low $\mathrm{T}_{3}$ ) is the most frequently observed alteration of thyroid hormone in CKD patients $(1,4)$. A recent study has shown that there is a significant correlation between serum $\mathrm{T}_{3}$ levels and estimated GFR (eGFR) and that the prevalence of low $\mathrm{T}_{3}$ gradually increases with worsening CKD stage (5). In addition, some previous studies have demonstrated that up to $70 \%$ of stage 5 CKD patients have low $\mathrm{T}_{3}(6)$. This alteration in CKD patients has mainly been attributed to impaired TSH response to thyrotropin-releasing hormone and decreased peripheral biosynthesis of $\mathrm{T}_{3}$ from $\mathrm{T}_{4}$ due to uremia and has been considered to be merely 
an adaptive process to preserve energy $(1,7)$. Recently, however, low $\mathrm{T}_{3}$ has also been suggested to be associated with metabolic acidosis, systemic inflammation, endothelial dysfunction, atherosclerosis, and cardiomyopathy in patients with end-stage renal disease (ESRD) $(8,9,10,11,12)$.

Accumulating evidence has indicated that low $\mathrm{T}_{3}$ is associated with clinical outcomes in patients with various chronic diseases, including congestive heart failure, respiratory failure, and liver cirrhosis (13, 14, $15,16)$. Since low $\mathrm{T}_{3}$ is the most common disturbance of thyroid hormone levels in ESRD patients, the adverse impact of low $\mathrm{T}_{3}$ on patient survival has been widely explored in patients on maintenance hemodialysis (HD) and peritoneal dialysis (PD) $(17,18)$. Furthermore, low pre-transplant serum $\mathrm{fT}_{3}$ levels have been revealed to be a significant predictor of subsequent graft failure in kidney transplant recipients (19). In spite of a large number of previous investigations on the prognostic value of low $\mathrm{T}_{3}$ for all-cause and cardiovascular $(\mathrm{CV})$ mortality in ESRD patients, the majority of the study subjects were prevalent $\mathrm{HD}$ or $\mathrm{PD}$ patients and echocardiographic findings were not included in the analysis in most of these studies. In addition, compared with $\mathrm{fT}_{3}$ concentrations, total $\mathrm{T}_{3}$ levels were more commonly determined in many hospitals and were less influenced by the way of measurement or medications such as heparin $(20,21,22)$, but clinical outcomes were more frequently defined based on serum or plasma $\mathrm{fT}_{3}$ concentrations than on total $\mathrm{T}_{3}$ concentrations.

Therefore, in this study, we determined whether low serum total $\mathrm{T}_{3}$ levels were a significant predictor of mortality in Korean incident HD patients from the Clinical Research Center for ESRD (CRC for ESRD) cohort. Moreover, the associations between $\mathrm{T}_{3}$ concentrations and metabolic acidosis, systemic inflammation, malnutrition, and echocardiographic parameters were investigated in these patients.

\section{Subjects and methods}

\section{Subjects}

Initial recruitment for this prospective observational multi-center study involved all ESRD patients starting HD between August 1, 2008, and October 31, 2012, at 36 centers of the CRC for ESRD in Korea. Within 7 days of the initial dialysis, the patients provided written informed consent and were enrolled into the study cohort. Among these patients, we excluded those who were aged $<18$ years, had a history of PD or kidney transplantation prior to HD, were on thyroid hormone replacement or suppressive therapy due to overt thyroid disease, were on medications affecting thyroid hormone levels, such as amiodarone, glucocorticoids, and lithium, had underlying active malignancy, or died within 3 months of the commencement of HD.
Patients who had insufficient baseline data, including thyroid function test results, were also excluded from the study. Ultimately, a total of 471 incident HD patients were included in the final analysis.

This study was carried out in accordance with the Declaration of Helsinki. The study protocol was approved by the Institutional Review Board at each participating center.

\section{Data collection}

Demographic and clinical data were recorded at the time of study entry, including age, gender, BMI calculated as weight/height ${ }^{2}$, primary renal disease, comorbidities, and medications. Coronary arterial disease (CAD) was defined as a history of angioplasty, coronary artery bypass grafts, myocardial infarction, or angina, while peripheral arterial disease (PAD) was defined as a history of claudication, ischemic limb loss and/or ulceration, or peripheral revascularization procedure. The composite of CAD, congestive heart failure of any cause ischemic, valvular disease related or amyloid deposition related to uremia, and arrhythmia was designated as cardiac disease, while the composite of cardiac disease, PAD, and cerebrovascular disease, transient ischemic attack, ischemia/infarction, or bleeding as cardiovascular disease (CVD). Laboratory data were measured using pre-dialysis fasting blood samples collected on the day of a mid-week dialysis session, when the patients were considered to be clinically stable and to be in euvolemic state. These laboratory data included data on hemoglobin (Hb), white blood cell (WBC) count, blood urea nitrogen, creatinine, calcium, phosphorus, intact parathyroid hormone, albumin, total cholesterol, triglyceride, alkaline phosphatase, HbA1c, sodium, potassium, bicarbonate, serum iron, ferritin, N-terminal pro-B-type natriuretic peptide (NT-proBNP), cardiac troponin $\mathrm{T}$ (cTnT), and high-sensitivity C-reactive protein (hs-CRP) levels. During follow-up, these data were measured every 3 months according to the K/DOQI guideline (http://www.kidney.org, June 26, 2013). eGFR was calculated using the four-variable Modification of Diet in Renal Disease (MDRD) Study and Chronic Kidney Disease Epidemiology Collaboration (CKD-EPI) Study equation (23). In addition, 24-h urine sample collection was executed to determine 24-h urinary protein, urea, and creatinine excretion. Nutritional status was also evaluated using subjective global assessment (SGA) score (24), lean body mass estimated by creatinine kinetics (LBM-Cr) (25), and normalized protein catabolic rate (nPCR) (26). Thyroid function test was performed 3 months after the initiation of $\mathrm{HD}$ and annually thereafter based on the consideration of the economic issue and the opinion of the clinician who participated in this prospective observational study. Serum $\mathrm{T}_{3}$, free $\mathrm{T}_{4}\left(\mathrm{fT}_{4}\right)$, and TSH levels were determined using a chemiluminescent microparticle immunoassay on the Architect-i2000SR analyzer (Abbott Laboratories), 
and the reference ranges were $0.58-1.59 \mathrm{ng} / \mathrm{ml}$, $0.70-1.48 \mathrm{ng} / \mathrm{dl}$, and $0.35-4.94 \mu \mathrm{IU} / \mathrm{ml}$ respectively. Since there was an extremely wide variation in the biochemical data at 0 month due to diverse patients' conditions to start $\mathrm{HD}$, we used the laboratory data collected at 3 months after HD commencement, when correction of uremia, anemia, and acidosis was considered to be sufficiently achieved.

Echocardiography was performed on a non-dialysis day, close to the time of discharge, based on the imaging protocol recommended by the American Society of Echocardiography. Left atrial dimension (LAD) was assessed at end-ventricular systole at the level of the aortic valve according to the leading-edge-to-leadingedge convention. Left ventricular (LV) mass was determined using the method described by Steiber et al. (24), and the LV mass index (LVMI) was calculated by dividing LV mass by body surface area. LV systolic function was defined by LV ejection fraction (LVEF) using a modified biplane Simpson method from the apical two-chamber and four-chamber views. Multiple reproducibility, inter-reader reliability, intra-reader reliability, and reader drift analyses were performed at a core echocardiography laboratory (Kyungpook National University, Daegu, Korea) on a random sample of $3 \%$ of the entire cohort each year. The intra-class correlation coefficients for the echocardiographic measures were 0.773 for LAD, 0.745 for LVMI, and 0.842 for LVEF.

\section{Outcome measures}

For this study, data on all mortality events were retrieved from the database and carefully reviewed. The primary and secondary endpoints were all-cause mortality and CV mortality respectively. CV mortality was considered death from myocardial infarction or ischemia, congestive heart failure, pulmonary edema, cerebral hemorrhage, or cerebrovascular disorder.

\section{Statistical analysis}

Statistical analysis was performed using SPSS for Windows, version 18.0 (SPSS, Inc.). Continuous variables are expressed as mean \pm s.D. or median (interquartile range) and categorical variables as a number (percentage). Normality of the distribution was ascertained using the Shapiro-Wilk test. The patients were dichotomized into 'higher' and 'lower' $\mathrm{T}_{3}$ groups based on the median values of serum $\mathrm{T}_{3}$ concentrations. Patient demographics, clinical characteristics, and laboratory findings were compared between the two groups using Student's $t$-test or Mann-Whitney $U$ test for continuous variables and the $\chi^{2}$ test for categorical variables. Because of the log-normal distributions of NT-proBNP, cTnT, and hs-CRP concentrations, natural $\log$ values (Ln) were used in the analysis. The associations of serum $\mathrm{T}_{3}$ levels with other variables were determined using Pearson's correlation analysis. A multivariate linear regression analysis was conducted to identify the independent determinants of $\mathrm{T}_{3}$ concentrations.

Cumulative survival curves for all-cause and CV mortality were created using the Kaplan-Meier method, and between-group survival was compared using a log-rank test. The independent prognostic power of serum $\mathrm{T}_{3}$ levels for mortality was ascertained using multivariate Cox proportional-hazards regression analysis, which included only the variables of $P$ values $<0.10$ in the univariate analysis. The positive predictive value of $\mathrm{T}_{3}$ concentrations for mortality was also analyzed using the receiver operating characteristic (ROC) curve analysis with calculated area under the ROC curve (AUC). $P$ values $<0.05$ were considered statistically significant.

\section{Results}

\section{Baseline characteristics}

The baseline demographic and clinical characteristics of the patients are given in Table 1 . The mean age was $56.9 \pm 14.9$ years and $57.3 \%$ of the patients were male. The most common cause of ESRD was diabetes (DM, 53.3\%), followed by hypertension (14.4\%). The median values of serum $\mathrm{T}_{3}, \mathrm{fT}_{4}$, and $\mathrm{TSH}$ concentrations were $0.8 \mathrm{ng} / \mathrm{ml}, 1.1 \mathrm{ng} / \mathrm{dl}$, and $1.9 \mu \mathrm{IU} / \mathrm{ml}$ respectively.

The demographic and clinical characteristics were compared between the dichotomized two groups based on the median levels of $\mathrm{T}_{3}(0.8 \mathrm{ng} / \mathrm{ml})$. The mean age, the proportion of female patients, and modified Charlson comorbidity index (CCI) were significantly higher in the 'lower' $\mathrm{T}_{3}$ group than in the 'higher' $\mathrm{T}_{3}$ group $(P<0.05$ or $<0.01)$. In addition, CVDs were significantly more prevalent in the 'lower' $\mathrm{T}_{3}$ group $(P=0.015)$. There was no significant difference in medication history between the two groups, except for the use of clopidogrel, which was prescribed more frequently in the 'lower' $\mathrm{T}_{3}$ group than in the 'higher' $\mathrm{T}_{3}$ group $(P<0.048)$.

With regard to the laboratory and echocardiographic findings, patients with 'lower' $\mathrm{T}_{3}$ levels demonstrated blood glucose and serum uric acid levels that were significantly higher, while $\mathrm{Hb}$, serum albumin, total cholesterol, triglyceride, and bicarbonate concentrations were significantly lower than those of the 'higher' $\mathrm{T}_{3}$ group $(P<0.05-P<0.001)$. However, there were no differences in serum calcium, phosphorus, NT-proBNP, cTnT, and hs-CRP levels and eGFR between the two groups. The proportion of malnourished patients assessed by SGA was significantly higher $(P=0.02)$ and LBM-Cr and nPCR were significantly lower in the 'lower' $\mathrm{T}_{3}$ group than in the 'higher' $\mathrm{T}_{3}$ group $(P<0.05)$. Among 379 patients $(192$ in the 'lower' $\mathrm{T}_{3}$ group and 187 in the 'higher' $\mathrm{T}_{3}$ group) who 
Table 1 Baseline demographic and clinical characteristics of the study subjects.

\begin{tabular}{|c|c|c|c|c|}
\hline Variables & Total $(n=471)$ & $\mathbf{T}_{3}<0.8 \mathrm{ng} / \mathrm{ml}(n=228)$ & $\mathbf{T}_{3} \geq 0.8 \mathrm{ng} / \mathrm{ml}(n=243)$ & $\boldsymbol{P}$ \\
\hline Age (years) & $56.85 \pm 14.94$ & $58.63 \pm 14.96$ & $55.19 \pm 14.76$ & 0.012 \\
\hline Sex (male) & $270(\overline{57} .3 \%)$ & $116(50.9 \%)$ & $154(63.4 \%)$ & 0.006 \\
\hline BMI $\left(\mathrm{kg} / \mathrm{m}^{2}\right)$ & $23.14 \pm 3.69$ & $22.71 \pm 3.89$ & $23.55 \pm 3.48$ & 0.065 \\
\hline Systolic BP (mmHg) & $143.0 \pm 23.4$ & $142.7 \pm 22.2$ & $143.3 \pm 24.5$ & 0.814 \\
\hline Diastolic BP (mmHg) & $77.6 \pm 14.0$ & $76.7 \pm 14.3$ & $78.5 \pm 13.6$ & 0.159 \\
\hline \multicolumn{4}{|l|}{ Smoking status } & \multirow{4}{*}{0.049} \\
\hline Current smoker & $54(11.5 \%)$ & $30(13.2 \%)$ & $24(9.9 \%)$ & \\
\hline Ex-smoker & $138(29.3 \%)$ & $55(24.1 \%)$ & $83(34.2 \%)$ & \\
\hline Non-smoker & $279(59.2 \%)$ & $143(62.7 \%)$ & $136(56.0 \%)$ & \\
\hline \multicolumn{4}{|l|}{ Primary cause of renal disease } & \multirow[t]{6}{*}{0.636} \\
\hline Diabetes & $251(53.3 \%)$ & $128(56.1 \%)$ & $123(50.6 \%)$ & \\
\hline Hypertension & $68(14.4 \%)$ & 35 (15.4\%) & $33(13.6 \%)$ & \\
\hline Chronic GN & $69(14.6 \%)$ & $29(12.7 \%)$ & $40(16.5 \%)$ & \\
\hline Others $^{a}$ & $50(10.6 \%)$ & $22(9.6 \%)$ & $28(11.5 \%)$ & \\
\hline Unknown & $33(7.0 \%)$ & $15(6.6 \%)$ & $18(7.4 \%)$ & \\
\hline \multicolumn{5}{|l|}{ Comorbid disease } \\
\hline Chronic lung disease & $47(10.0 \%)$ & $25(11.0 \%)$ & $22(9.1 \%)$ & 0.497 \\
\hline Coronary arterial disease & $72(15.3 \%)$ & 40 (17.5\%) & $32(13.2 \%)$ & 0.186 \\
\hline Peripheral arterial disease & $47(10.0 \%)$ & $27(11.8 \%)$ & $20(8.2 \%)$ & 0.195 \\
\hline Cerebrovascular disease & $62(13.2 \%)$ & $36(15.8 \%)$ & $26(10.7 \%)$ & 0.103 \\
\hline Congestive heart failure & $73(15.5 \%)$ & $42(18.4 \%)$ & $31(12.8 \%)$ & 0.093 \\
\hline Arrhythmia & $13(2.8 \%)$ & $7(3.1 \%)$ & $6(2.5 \%)$ & 0.690 \\
\hline Diabetes & $260(55.2 \%)$ & $131(57.5 \%)$ & $129(53.1 \%)$ & 0.366 \\
\hline Connective tissue disease & $50(10.6 \%)$ & $24(10.5 \%)$ & $26(10.7 \%)$ & 0.941 \\
\hline Ulcer & $42(8.9 \%)$ & $21(9.2 \%)$ & $21(8.6 \%)$ & 0.827 \\
\hline Liver disease & 47 (10.0\%) & $19(8.3 \%)$ & $26(10.7 \%)$ & 0.383 \\
\hline Cardiac disease $^{\mathrm{b}}$ & $121(25.7 \%)$ & $68(29.8 \%)$ & $53(21.8 \%)$ & 0.047 \\
\hline CVD $^{\mathrm{C}}$ & $172(36.5 \%)$ & $96(42.1 \%)$ & $76(31.3 \%)$ & 0.015 \\
\hline Modified CCI & $5.16 \pm 2.45$ & $5.44 \pm 2.52$ & $4.90 \pm 2.36$ & 0.017 \\
\hline \multicolumn{5}{|l|}{ Medications } \\
\hline RAS blockers & 241 (51.2\%) & $123(53.9 \%)$ & $118(48.6 \%)$ & 0.242 \\
\hline $\mathrm{CCB}$ & 268 (56.9\%) & 127 (55.7\%) & $141(58.0 \%)$ & 0.611 \\
\hline Beta blocker & $251(53.3 \%)$ & $114(50.0 \%)$ & $137(56.4 \%)$ & 0.166 \\
\hline Aspirin & $106(22.5 \%)$ & $58(25.4 \%)$ & $48(19.8 \%)$ & 0.140 \\
\hline Clopidogrel & $34(7.2 \%)$ & $22(9.6 \%)$ & $12(4.9 \%)$ & 0.048 \\
\hline Vitamin D & $64(13.6 \%)$ & $35(15.4 \%)$ & $29(11.9 \%)$ & 0.280 \\
\hline ESA & 159 (33.8\%) & $69(30.3 \%)$ & $90(37.0 \%)$ & 0.120 \\
\hline
\end{tabular}

BP, blood pressure; GN, glomerulonephritis; CVD, cardiovascular disease; CCI, Charlson comorbidity index; RAS, renin-angiotensin system; CCB, calcium channel blocker; ESA, erythropoietin-stimulating agent.

${ }^{a}$ Other causes of renal failure include polycystic kidney disease, interstitial nephritis, acquired obstructive uropathy, chronic pyelonephritis, reflux nephropathy, and amyloidosis.

${ }^{b}$ Composite of coronary arterial disease, congestive heart failure, and arrhythmia.

${ }^{\mathrm{C}}$ Composite of coronary arterial disease, congestive heart failure, arrhythmia, peripheral arterial disease, and cerebrovascular disease.

underwent echocardiography, the 'lower' $\mathrm{T}_{3}$ group had a significantly higher LVMI $(P=0.002)$ and a lower LVEF $(P=0.012)$ than the 'higher' $\mathrm{T}_{3}$ group (Table 2).

\section{Associations of $\mathrm{T}_{3}$ levels with malnutrition and inflammation markers and echocardiographic findings}

Pearson's correlation analysis revealed that serum $\mathrm{T}_{3}$ concentrations had significant inverse correlations with age $(r=-0.156, P=0.001)$, serum glucose levels $(r=-0.180, P<0.001)$, WBC counts $(r=-0.114$, $P=0.016)$, and Ln hs-CRP $(r=-0.150, P=0.003)$. In contrast, there were significant positive associations between serum $\mathrm{T}_{3}$ concentrations and serum bicarbonate $(r=0.234, P<0.001)$, albumin $(r=0.354$, $P<0.001)$, total cholesterol $(r=0.179, P=0.001)$, and triglyceride $(r=0.193, P<0.001)$ levels, LBM-Cr $(r=0.174, P=0.003)$, and nPCR $(r=0.223, P<0.001)$. Furthermore, serum $\mathrm{T}_{3}$ concentrations had a significant inverse correlation with LVMI $(r=-0.157$, $P=0.002)$ and a significant positive relationship with LVEF ( $r=0.168, P=0.001)$ (Table 3 and Fig. 1 ).

Furthermore, a significant association of serum $\mathrm{T}_{3}$ levels with serum albumin $(r=0.244, P=0.002)$ and bicarbonate $(r=0.348, P<0.001)$ concentrations still persisted in 163 patients even at 12 months (Supplementary Figure 1, see section on supplementary data given at the end of this article).

\section{Determinants of serum $T_{3}$ levels}

The multivariate linear regression analysis, including variables with $P$ values $<0.1$ in the univariate analysis, demonstrated that age, serum albumin, bicarbonate, triglyceride, and hs-CRP concentrations, and nPCR 
Table 2 Laboratory and echocardiographic findings of the study subjects.

\begin{tabular}{|c|c|c|c|c|}
\hline Variables & Total $(n=471)$ & $\mathbf{T}_{3}<0.8 \mathrm{ng} / \mathrm{ml}(n=228)$ & $\mathbf{T}_{3} \geq 0.8 \mathrm{ng} / \mathrm{ml}(n=243)$ & $\boldsymbol{P}$ \\
\hline \multicolumn{5}{|l|}{ Laboratory data at 3 months } \\
\hline Hemoglobin $(\mathrm{g} / \mathrm{dl})$ & $10.84 \pm 1.41$ & $10.58 \pm 1.32$ & $11.01 \pm 1.45$ & 0.001 \\
\hline WBC $(/ \mu \mathrm{l})$ & $5883.4 \pm 2996.4$ & $5911.4 \pm 3040.1$ & $5282.5 \pm 3106.5$ & 0.032 \\
\hline Calcium (mg/dl) & $8.67 \pm 0.81$ & $8.69 \pm 0.87$ & $8.63 \pm 0.76$ & 0.731 \\
\hline Phosphorus (mg/dl) & $4.70 \pm 1.44$ & $4.71 \pm 1.43$ & $4.70 \pm 1.45$ & 0.909 \\
\hline Uric acid (mg/dl) & $6.91 \pm 1.96$ & $7.10 \pm 1.93$ & $6.74 \pm 1.98$ & 0.043 \\
\hline $\mathrm{iPTH}(\mathrm{pg} / \mathrm{ml})$ & $182.59 \pm 54.27$ & $180.45 \pm 47.18$ & $184.57 \pm 60.93$ & 0.789 \\
\hline Glucose $(\mathrm{mg} / \mathrm{dl})$ & $124.17 \pm 44.24$ & $137.77 \pm 55.46$ & $111.58 \pm 40.73$ & 0.003 \\
\hline $\mathrm{HbA1c}(\%)$ & $6.10 \pm 1.41$ & $6.14 \pm 1.50$ & $6.06 \pm 1.29$ & 0.453 \\
\hline Protein $(\mathrm{g} / \mathrm{dl})$ & $6.64 \pm 0.73$ & $6.49 \pm 0.71$ & $6.76 \pm 0.73$ & $<0.001$ \\
\hline Albumin (g/dl) & $3.76 \pm 0.52$ & $3.61 \pm 0.52$ & $3.89 \pm 0.47$ & $<0.001$ \\
\hline BUN (mg/dl) & $54.89 \pm 21.38$ & $56.56 \pm 19.64$ & $53.27 \pm 22.92$ & 0.258 \\
\hline Creatinine $(\mathrm{mg} / \mathrm{dl})$ & $7.75 \pm 3.16$ & $7.84 \pm 3.19$ & $7.67 \pm 2.98$ & 0.293 \\
\hline GFR-MDRD $\left(\mathrm{ml} / \mathrm{min}\right.$ per $\left.1.73 \mathrm{~m}^{2}\right)$ & $6.92 \pm 3.71$ & $6.86 \pm 2.94$ & $6.99 \pm 4.38$ & 0.187 \\
\hline GFR-EPI $\left(\mathrm{ml} / \mathrm{min}\right.$ per $\left.1.73 \mathrm{~m}^{2}\right)$ & $7.14 \pm 3.74$ & $7.04 \pm 2.95$ & $7.23 \pm 4.42$ & 0.218 \\
\hline Total cholesterol (mg/dl) & $153.20 \pm 36.69$ & $147.05 \pm 37.40$ & $158.99 \pm 35.71$ & 0.019 \\
\hline Triglyceride (mg/dl) & $126.01 \pm 46.49$ & $116.77 \pm 41.84$ & $134.12 \pm 54.70$ & 0.012 \\
\hline Sodium $(\mathrm{mmol} / \mathrm{l})$ & $138.11 \pm 4.17$ & $137.83 \pm 4.77$ & $138.35 \pm 3.50$ & 0.067 \\
\hline Potassium (mmol/l) & $4.73 \pm 0.83$ & $4.74 \pm 0.79$ & $4.72 \pm 0.87$ & 0.726 \\
\hline Bicarbonate $(\mathrm{mmol} / \mathrm{l})$ & $22.47 \pm 4.41$ & $21.80 \pm 4.39$ & $23.12 \pm 4.35$ & 0.007 \\
\hline hs-CRP (mg/dl) & $0.14(0.04-0.55)$ & $0.16(0.04-0.74)$ & $0.13(0.04-0.50)$ & 0.167 \\
\hline NT-proBNP (pg/ml) & $7377.0(2074.0-26328.0)$ & $8613.0(2124.0-29821.5)$ & $5385.5(1940.3-18780.0)$ & 0.107 \\
\hline cTnT $(\mathrm{ng} / \mathrm{ml})$ & $0.054(0.028-0.113)$ & $0.060(0.030-0.126)$ & $0.047(0.026-0.093)$ & 0.110 \\
\hline $\mathrm{T}_{3}(\mathrm{ng} / \mathrm{ml})$ & $0.80 \pm 0.28$ & $0.58 \pm 0.16$ & $1.00 \pm 0.19$ & $<0.001$ \\
\hline Free $T_{4}(n g / d l)$ & $1.10 \pm 0.26$ & $1.08 \pm 0.24$ & $1.12 \pm 0.28$ & 0.084 \\
\hline TSH $(\mu \mathrm{IU} / \mathrm{ml})$ & $1.90(1.24-3.32)$ & $1.90(1.20-3.29)$ & $1.94(1.24-3.35)$ & 0.899 \\
\hline \multicolumn{5}{|l|}{ 24-h urine study } \\
\hline Urea $(\mathrm{mg} / \mathrm{dl})$ & $247.6(158.9-451.7)$ & $238.0(158.1-353.0)$ & $261.5(163.8-970.0)$ & 0.129 \\
\hline Creatinine (mg/dl) & $68.2(42.6-181.4)$ & $64.6(39.3-114.9)$ & $76.9(44.9-327.2)$ & 0.017 \\
\hline Protein (mg/day) & $922.4(349.2-2109.1)$ & $772.2(348.4-1767.4)$ & $1056.0(353.7-2461.7)$ & 0.124 \\
\hline Urine volume (ml/day) & $780.0(460.0-1100.0)$ & $700.0(400.0-1022.5)$ & $800.0(490.0-1150.0)$ & 0.118 \\
\hline \multicolumn{5}{|l|}{ Nutritional markers } \\
\hline SGA $>1$ (malnutrition) & $147(31.2 \%)$ & $83(36.4 \%)$ & $64(26.3 \%)$ & 0.020 \\
\hline LBM-Cr & $44.89 \pm 6.08$ & $44.20 \pm 6.05$ & $45.72 \pm 6.05$ & 0.033 \\
\hline $\mathrm{nPCR}$ & $0.94 \pm 0.39$ & $0.86 \pm 0.34$ & $1.05 \pm 0.46$ & 0.021 \\
\hline $\mathrm{nPCR} \geq 1.0$ & $118(25.1 \%)$ & $52(22.8 \%)$ & $66(27.2 \%)$ & 0.007 \\
\hline \multicolumn{5}{|c|}{ Baseline echocardiographic parameters } \\
\hline LAD $(\mathrm{cm})$ & $4.30 \pm 0.74$ & $4.28 \pm 0.74$ & $4.33 \pm 0.74$ & 0.476 \\
\hline LVMI $\left(\mathrm{g} / \mathrm{m}^{2}\right)$ & $144.05 \pm 49.91$ & $151.71 \pm 56.54$ & $136.04 \pm 40.52$ & 0.002 \\
\hline LVEF (\%) & $58.66 \pm 11.31$ & $57.22 \pm 12.44$ & $60.13 \pm 9.83$ & 0.012 \\
\hline
\end{tabular}

WBC, white blood cell; iPTH, intact parathyroid hormone; BUN, blood urea nitrogen; GFR, glomerular filtration rate; MDRD, Modification of Diet in Renal Disease; EPI, Chronic Kidney Disease Epidemiology Collaboration equation; hs-CRP, high-sensitivity C-reactive protein; NT-proBNP, N-terminal pro-B-type natriuretic peptide; cTnT, cardiac troponin T; SGA, subjective global assessment; LBM-Cr, lean body mass estimated by creatinine kinetics; nPCR, normalized protein catabolic rate; LAD, left atrial diameter; LVMI, left ventricular mass index; LVEF, left ventricular ejection fraction.

were significant independent determinants of serum $\mathrm{T}_{3}$ levels (Table 4).

\section{Clinical outcomes according to serum $T_{3}$ concentrations}

During a mean follow-up duration of $24.2 \pm 17.7$ months, 49 patients $(10.4 \%)$ died. Among them, 22 patients $(44.9 \%)$ died from CV causes and $12(24.5 \%)$ from infection. All-cause, CV, and infection-related mortality rates were significantly higher in patients with 'lower' $\mathrm{T}_{3}$ levels than in the 'higher' $\mathrm{T}_{3}$ group (all-cause mortality: 113.4 vs 18.2 events per 1000 patient-years, $P<0.001$; CV mortality: 49.8 vs 9.1 events per 1000 patient-years, $P=0.001$; and infectionrelated mortality: 27.7 vs 4.5 events per 1000 patientyears, $P=0.014$ ) (Table 5). The Kaplan-Meier analysis also indicated that the 'lower' $\mathrm{T}_{3}$ group had significantly worse all-cause and $\mathrm{CV}$ cumulative survival rates compared with the 'higher' $\mathrm{T}_{3}$ group $(P<0.001$ and $P=0.001$ respectively) (Fig. 2). Overall hospitalization rates, as well as composite death and hospitalization rates, were also significantly higher in patients with 'lower' $\mathrm{T}_{3}$ levels than in the 'higher' $\mathrm{T}_{3}$ group (Table 5).

On the other hand, serum $\mathrm{T}_{3}$ levels provided significant positive predictive values for all-cause mortality before (AUC $=0.674, P=0.005)$ and even after adjusting for BMI, smoking status, modified CCI, aspirin and clopidogrel usage, serum $\mathrm{HbAlc}$, albumin, and potassium concentrations, eGFR by CKD-EPI, Ln hs-CRP, and SGA score $(\mathrm{AUC}=0.878, \mathrm{P}<0.001)$ (Fig. 3). There was also a significant positive predictive value of $\mathrm{T}_{3}$ levels for $\mathrm{CV}$ death $(\mathrm{AUC}=0.702, P=0.007)$ (data not shown). 
Table 3 Correlations of serum $\mathrm{T}_{3}$ levels with various nutritional, inflammatory, and echocardiographic variables.

\begin{tabular}{|c|c|c|}
\hline \multirow[b]{2}{*}{ Variables } & \multicolumn{2}{|c|}{$\mathrm{T}_{3}(\mathrm{ng} / \mathrm{ml})$} \\
\hline & $r$ & $P$ \\
\hline Age (years) & -0.156 & 0.001 \\
\hline Sex (male) & -0.162 & $<0.001$ \\
\hline Glucose & -0.180 & $<0.001$ \\
\hline $\mathrm{HbA} 1 \mathrm{c}(\%)$ & -0.042 & 0.432 \\
\hline Bicarbonate (mmol/l) & 0.234 & $<0.001$ \\
\hline \multicolumn{3}{|l|}{ Nutritional markers } \\
\hline $\mathrm{BMI}\left(\mathrm{kg} / \mathrm{m}^{2}\right)$ & 0.076 & 0.104 \\
\hline Albumin (g/dl) & 0.354 & $<0.001$ \\
\hline Total cholesterol & 0.179 & 0.001 \\
\hline Triglyceride & 0.193 & $<0.001$ \\
\hline LBM-Cr & 0.174 & 0.003 \\
\hline nPCR & 0.223 & $<0.001$ \\
\hline \multicolumn{3}{|l|}{ Inflammatory markers } \\
\hline White blood cell $(/ \mu \mathrm{l})$ & -0.114 & 0.016 \\
\hline Ln hs-CRP & -0.150 & 0.003 \\
\hline \multicolumn{3}{|c|}{ Echocardiographic parameters } \\
\hline $\mathrm{LAD}(\mathrm{cm})$ & 0.001 & 0.978 \\
\hline LVMI $\left(\mathrm{g} / \mathrm{m}^{2}\right)$ & -0.157 & 0.002 \\
\hline LVEF (\%) & 0.168 & 0.001 \\
\hline
\end{tabular}

LBM-Cr, lean body mass estimated by creatinine kinetics; nPCR, normalized protein catabolic rate; Ln hs-CRP, log-transformed high-sensitivity C-reactive protein; LAD, left atrial diameter; LVMI, left ventricular mass index; LVEF, left ventricular ejection fraction.

\section{Low $\mathrm{T}_{3}$ as a predictor of all-cause mortality}

The univariate Cox proportional-hazards regression analysis revealed that BMI, smoking, modified CCI, aspirin and clopidogrel usage, eGFR by CKD-EPI, serum HbA1c, albumin, and potassium concentrations, Ln hs-CRP, LBM-Cr, nPCR <1.0, LVMI, LVEF, and low $\mathrm{T}_{3}$ were significant risk factors for all-cause mortality (Supplementary Table 1, see section on supplementary data given at the end of this article). In multivariate
Cox models, low $\mathrm{T}_{3}$ remained as a significant independent predictor of all-cause mortality (hazard ratio $(\mathrm{HR})=3.758,95 \% \quad \mathrm{CI}=1.055-13.382, P=0.021)$, even after adjusting for BMI, smoking status, modified CCI, aspirin and clopidogrel usage, eGFR by CKD-EPI, serum HbA1c, albumin, and potassium levels, Ln hs-CRP, and SGA score. However, when LBM-Cr, nPCR, LVMI, or LVEF were included in the successive models for further adjustment, the significant impact of low $\mathrm{T}_{3}$ on all-cause mortality disappeared (Table 6).

In another analysis, we divided the patients into 'subnormal' and 'normal' $\mathrm{T}_{3}$ groups based on the lowest normal value of $\mathrm{T}_{3}(0.58 \mathrm{ng} / \mathrm{ml})$ and determined the impact of 'subnormal' $\mathrm{T}_{3}$ on the clinical outcome. Similarly, 'subnormal' $\mathrm{T}_{3}$ was a significant risk factor for all-cause mortality $(\mathrm{HR}=5.943$, 95\% CI $=1.442$ 24.492, $P=0.014)$, but its statistical power disappeared when LBM-Cr, nPCR, LVMI, or LVEF were further incorporated into the models (Supplementary Tables 2 and 3, see section on supplementary data given at the end of this article).

Furthermore, low $\mathrm{T}_{3}$ was not a significant independent predictor of $\mathrm{CV}$ mortality after adjusting for smoking status, DM, underlying CVDs, eGFR by CKD-EPI, serum albumin and potassium concentrations, Ln hs-CRP, and SGA score $(\mathrm{HR}=2.735$, $95 \% \mathrm{CI}=0.917-11.395, P=0.086)$ (data not shown).

\section{Discussion}

Thyroid hormone abnormalities are common in CKD patients. Among these, low $\mathrm{T}_{3}$ is the most frequently observed alteration of thyroid hormone in patients with CKD. Recently, accumulating evidence has indicated that low $\mathrm{T}_{3}$ may act as a prognostic factor for adverse clinical outcomes in prevalent ESRD patients rather
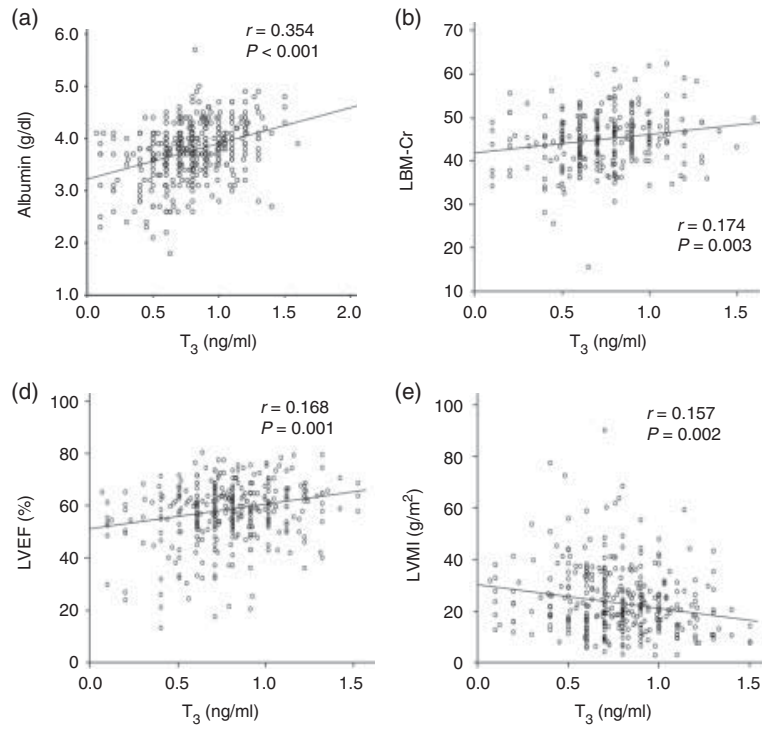
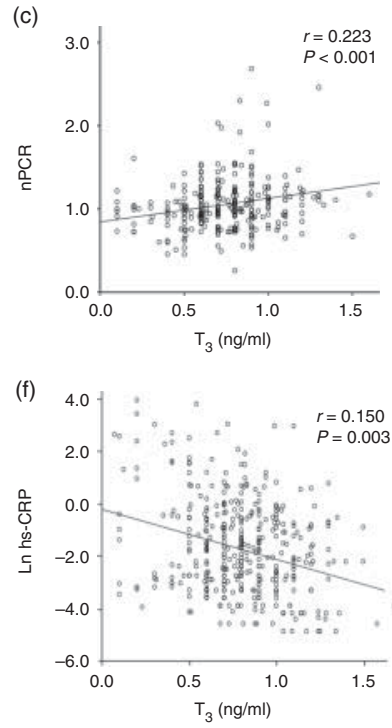

Figure 1 The correlation of serum $\mathrm{T}_{3}$ levels with various nutritional, inflammatory, and echocardiographic variables. Serum $\mathrm{T}_{3}$ concentrations had significant positive associations with (a) serum albumin levels, (b) LBM-Cr,

(c) nPCR, and (d) LVEF, while there were significant inverse correlations of $T_{3}$ with (e) LVMI and (f) Ln hs-CRP. LBM-Cr, lean body mass estimated by creatinine kinetics; $\mathrm{nPCR}$, normalized protein catabolic rate; LVEF, left ventricular ejection fraction; LVMI, left ventricular mass index; Ln hs-CRP, log-transformed high-sensitivity $\mathrm{C}$-reactive protein. 
Table 4 Determinants of serum $\mathrm{T}_{3}$ concentrations.

\begin{tabular}{lrc}
\hline & \multicolumn{2}{c}{$\mathbf{T}_{\mathbf{3}}$} \\
\cline { 2 - 3 } Variables & $\begin{array}{c}\text { Regression } \\
\text { coefficient }\end{array}$ \\
\hline Age (years) & -0.003 & 0.017 \\
Sex (male vs female) & 0.025 & 0.448 \\
Underlying cardiovascular disease & 0.027 & 0.424 \\
Hemoglobin (g/dl) & 0.009 & 0.489 \\
Glucose (mg/dl) & -0.022 & 0.749 \\
Albumin (g/dl) & 0.068 & 0.047 \\
Total cholesterol (mg/dl) & 0.003 & 0.983 \\
Triglyceride (mg/dl) & 0.179 & 0.013 \\
Bicarbonate (mmol/l) & 0.010 & 0.006 \\
SGA $>1$ (vs SGA $\leq 1)$ & -0.033 & 0.354 \\
nPCR $\geq 1.0(\mathrm{vs} \mathrm{nPCR}<1.0)$ & 0.107 & 0.001 \\
hs-CRP $\geq 5 \mathrm{mg} / \mathrm{dl}(\mathrm{vs} \mathrm{hs-CRP}<5 \mathrm{mg} / \mathrm{dl})$ & -0.192 & 0.019 \\
\hline
\end{tabular}

SGA, subjective global assessment; nPCR, normalized protein catabolic rate; hs-CRP, high-sensitivity C-reactive protein.

than as an innocent bystander $(17,18)$. In this study, we demonstrated that low $\mathrm{T}_{3}$ is a significant independent risk factor for all-cause mortality in incident $\mathrm{HD}$ patients. Moreover, it seems that the impact of low $\mathrm{T}_{3}$ on mortality may be due, in part, to malnutrition and cardiac dysfunction.

Low $\mathrm{T}_{3}$ has been reported to be present in up to $70 \%$ of patients with $\operatorname{ESRD}(5,6)$. It has been proposed that multiple mechanisms contribute to this high prevalence of low $\mathrm{T}_{3}$, including systemic inflammation, malnutrition, and metabolic acidosis $(8,9)$. Zoccali et al. (17) showed that $\mathrm{fT}_{3}$ was a significant independent predictor of mortality even after adjusting for a series of traditional and non-traditional risk factors including interleukin-6 (IL6) in a multivariate analysis, but that this inflammatory cytokine predicted mortality only in a model excluding $\mathrm{fT}_{3}$. These findings suggest that $\mathrm{fT}_{3}$ and inflammation are involved in the same pathogenic pathway leading to death in ESRD patients and that $\mathrm{fT}_{3}$ mediates part of the adverse effects of inflammation in these patients. Moreover, similar findings have been demonstrated in prevalent PD patients (18). We also found that plasma $T_{3}$ levels were significantly associated with hs-CRP concentrations and that hs-CRP was a significant independent determinant of $\mathrm{T}_{3}$. In contrast to a majority of previous studies, however, there was no difference in hs-CRP levels between the 'lower' and 'higher' $\mathrm{T}_{3}$ groups in the present study. It is surmised that this discrepancy may partly be due to differing ethnicities and dialysis duration (prevalent vs incident). Further investigation, including various inflammatory markers in a large, ethnically homogenous incident HD patient cohort, will be needed to confirm whether the impact of $\mathrm{T}_{3}$ levels on mortality is independent or associated with inflammation.

It is well known that $\mathrm{T}_{3}$ increases cardiac output by affecting tissue oxygen consumption, vascular resistance, blood volume, cardiac contractility, and heart rate $(28,29)$. Therefore, low $\mathrm{T}_{3}$, even in the normal range, has been suggested to be associated with various CVDs in ESRD patients, which has been clarified by several previous studies $(12,17,30,31)$. In their study, Zoccali et al. (12) showed that LV systolic function was depressed and LVMI was increased in the first $\mathrm{fT}_{3}$ quartile of 234 prevalent dialysis patients when compared with patients in other quartiles. In another recent, cross-sectional study, across decreasing $\mathrm{fT}_{3}$ tertiles, carotid-femoral pulse wave velocity (c-f PWV) and carotid artery-intima media thickness (CA-IMT) measured by Doppler ultrasonography were found to be incrementally higher in 137 maintenance HD patients. Furthermore, $\mathrm{fT}_{3}$ concentrations have been demonstrated to be significantly associated with c-f PWV and CA-IMT, indicating that $\mathrm{fT}_{3}$ levels are negatively associated with carotid atherosclerosis and arterial stiffness (30). The present study also found that in the 'lower' $\mathrm{T}_{3}$ group, LVEF was significantly lower, whereas LVMI was significantly higher, which was in concordance with previous observations. In addition,

Table 5 Comparisons of clinical outcomes according to serum $T_{3}$ levels.

\begin{tabular}{|c|c|c|c|c|c|}
\hline & \multicolumn{2}{|c|}{$\mathbf{T}_{\mathbf{3}}<0.8 \mathrm{ng} / \mathrm{ml}(n=228)$} & \multicolumn{2}{|c|}{$\mathbf{T}_{3} \geq 0.8 \mathrm{ng} / \mathrm{ml}(n=243)$} & \multirow[b]{2}{*}{$\boldsymbol{P}$} \\
\hline & $n(\%)$ & $\begin{array}{c}\text { Rates (per } 1000 \\
\text { patient-years) }\end{array}$ & $n(\%)$ & $\begin{array}{c}\text { Rates (per } 1000 \\
\text { patient-years) }\end{array}$ & \\
\hline \multicolumn{6}{|l|}{ Mortality } \\
\hline All-cause & $41(18.0 \%)$ & 113.36 & 8 (3.3\%) & 18.15 & $<0.001$ \\
\hline CV & $18(7.9 \%)$ & 49.77 & $4(1.6 \%)$ & 9.08 & 0.001 \\
\hline Infection & $10(4.4 \%)$ & 27.65 & $2(0.8 \%)$ & 4.54 & 0.014 \\
\hline \multicolumn{6}{|c|}{ Hospitalization } \\
\hline All-cause & $102(44.7 \%)$ & 282.07 & 75 (30.9\%) & 170.16 & 0.002 \\
\hline CV & $35(15.4 \%)$ & 96.77 & $24(9.9 \%)$ & 54.45 & 0.073 \\
\hline Infection & $29(12.7 \%)$ & 80.18 & $14(5.8 \%)$ & 31.76 & 0.009 \\
\hline \multicolumn{6}{|c|}{ Composite $^{a}$} \\
\hline All-cause & $132(57.9 \%)$ & 364.96 & $79(32.5 \%)$ & 179.24 & $<0.001$ \\
\hline CV & $48(21.1 \%)$ & 132.71 & $24(9.9 \%)$ & 54.45 & 0.001 \\
\hline Infection & $33(14.5 \%)$ & 91.24 & $14(5.8 \%)$ & 31.76 & 0.002 \\
\hline
\end{tabular}



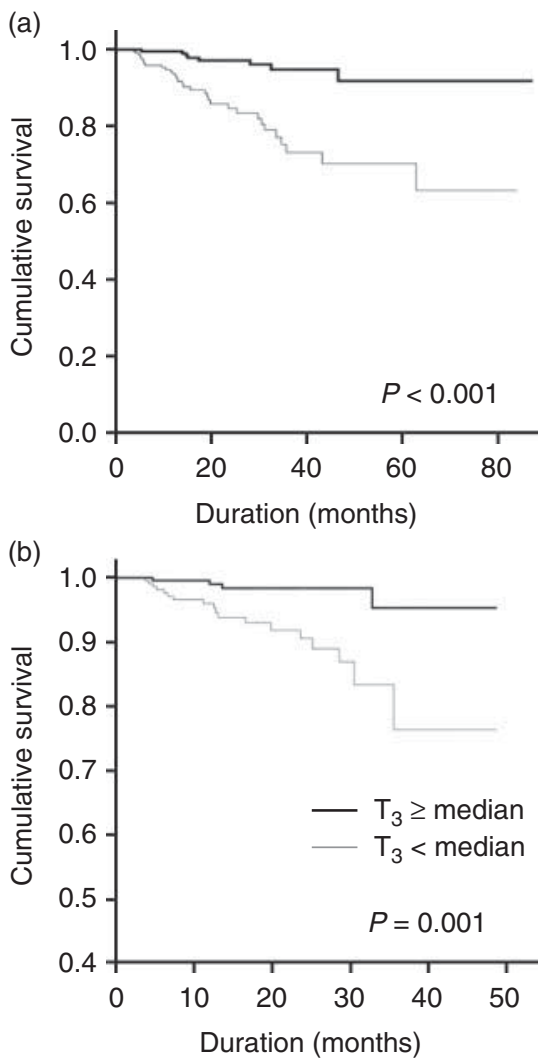

Figure 2 Kaplan-Meier plots for all-cause and cardiovascular survival. Compared with patients with 'higher' $T_{3}$, the 'lower' $T_{3}$ group showed significantly worse (a) all-cause and (b) cardiovascular cumulative survival rates.

serum $\mathrm{T}_{3}$ concentrations had a significant inverse correlation with LVMI and a significant positive relationship with LVEF. Even though a multivariate Cox hazards regression analysis revealed that low $\mathrm{T}_{3}$ was a significant risk factor for mortality after adjusting for traditional risk factors, the independent predictive value of low $\mathrm{T}_{3}$ for mortality disappeared when LVEF and/or LVMI were included in a multivariate Cox model. These findings suggest that the association between $\mathrm{T}_{3}$ levels and all-cause mortality is largely mediated by cardiac dysfunction. Similarly, cardiac abnormalities, low LVEF and high LVMI had a significant impact on allcause mortality only when $T_{3}$ levels were excluded from the multivariate analysis (data not shown), suggesting that the relationship between cardiac dysfunction and mortality was mediated by $\mathrm{T}_{3}$.

A recent study by Ozen et al. (6) has shown that $\mathrm{fT}_{3}$ is a significant predictor of mortality in adjusted Cox regression models including albumin or hs-CRP levels, but that the significance is less when compared with that of a crude Cox model. Moreover, further adjustment for both albumin and hs-CRP levels made the impact of $\mathrm{fT}_{3}$ levels on mortality disappear. These findings suggest that $\mathrm{fT}_{3}$ is a strong and inverse mortality predictor, in part explained by its underlying association with nutritional state and inflammation. In this study, we included for the first time not only albumin levels but also the SGA score, LBM-Cr, and nPCR to determine the relationship between $\mathrm{T}_{3}$ and nutritional status and demonstrated that $\mathrm{T}_{3}$ levels were significantly associated with nutritional parameters in incident HD patients. Furthermore, $\mathrm{T}_{3}$ was a significant independent predictor of allcause mortality in a traditional risk factors-adjusted Cox model. However, the relationship became insignificant when nutritional parameters were included in the multivariate Cox hazards regression analysis. Similarly, the impact of nutritional parameters on mortality was no longer significant when $\mathrm{T}_{3}$ was included in the Cox model (data not shown). Taken together, the prognostic power of $\mathrm{T}_{3}$ and nutrition seem to be interrelated.

Several aspects of the methods and scope of this study may limit the interpretation of research outcomes. First, since the study subjects were all Korean incident HD
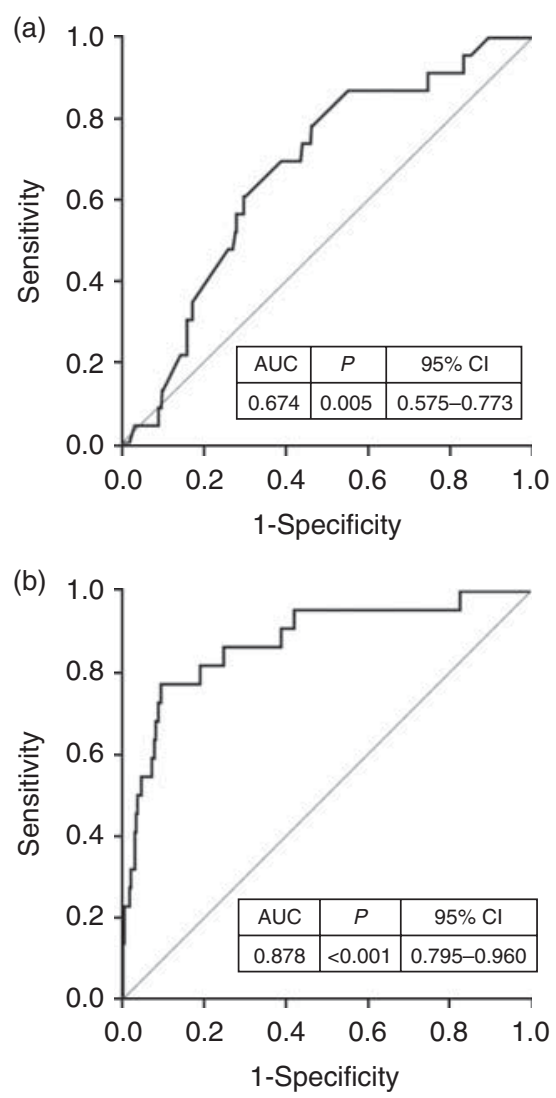

Figure 3 Receiver operating characteristic curves of $T_{3}$ for all-cause mortality. Serum $\mathrm{T}_{3}$ concentrations provided significant positive predictive values for all-cause mortality (a) in an unadjusted model $(A \cup C=0.674)$ and $(b)$ in a model adjusted for BMI, smoking status, modified $\mathrm{CCl}$, aspirin and clopidogrel usage, serum $\mathrm{HbA} 1 \mathrm{c}$, albumin, and potassium concentrations, eGFR by CKD-EPI, $\mathrm{Ln}$ hs-CRP, and SGA score $(\mathrm{AUC}=0.878)$. CCl, Charlson comorbidity index; eGFR, estimated glomerular filtration rate; CKDEPI, Chronic Kidney Disease Epidemiology Collaboration; Ln hs-CRP, log-transformed high-sensitivity C-reactive protein; SGA, subjective global assessment. 
Table 6 Multivariate Cox proportional-hazards regression analysis for all-cause mortality.

\begin{tabular}{lccc}
\hline & \multicolumn{3}{c}{ Serum $\mathrm{T}_{\mathbf{3}}$} \\
\cline { 2 - 4 } & $\mathrm{HR}$ & $95 \% \mathrm{Cl}$ & $P$ \\
\hline Model 1 & 3.758 & $1.055-13.382$ & 0.021 \\
Model 2 & 7.715 & $0.976-60.987$ & 0.053 \\
Model 3 & 7.692 & $0.973-60.816$ & 0.052 \\
Model 4 & 6.860 & $0.839-56.096$ & 0.072 \\
Model 5 & 8.008 & $0.971-66.046$ & 0.053 \\
\hline
\end{tabular}

Model 1: adjusted for BMI, smoking status, modified Charlson comorbidity index, aspirin and clopidogrel usage, glomerular filtration rate estimated by Chronic Kidney Disease Epidemiology Collaboration equation, serum $\mathrm{HbA1c}$ albumin, and potassium concentrations, log-transformed high-sensitivity C-reactive protein, and subjective global assessment score; Model 2: Mode $1+$ lean body mass estimated by creatinine kinetics; Model 3: Model $1+$ normalized protein catabolic rate $\geq 1.0$ (vs $<1.0$ ); Model 4: Model $1+$ left ventricular mass index; Model 5: Model $1+$ left ventricular ejection fraction.

patients, the association between $\mathrm{T}_{3}$ levels and mortality may not be generalized to other populations. Second, we focused on studying the effect of $\mathrm{T}_{3}$ levels in this study, while previous investigations have found that both low $\mathrm{T}_{3}$ and $\mathrm{fT}_{3}$ levels are independent risk factors for mortality in prevalent dialysis patients $(6,17,18,32)$. In incident dialysis patients, however, only $\mathrm{T}_{3}$, but not $\mathrm{fT}_{3}$, levels were associated with worse all-cause and CV mortality after adjustment for confounding factors (31). This may be attributed to a lower technical specificity of $\mathrm{fT}_{3}$ measurement by RIA (20), especially for the stored samples (21), and a smaller influence of heparin on $\mathrm{T}_{3}$ than on $\mathrm{fT}_{3}(22,33)$. In addition, $\mathrm{T}_{3}$ levels are determined as a total form rather than as a free form in most hospitals in Korea due to the financial benefits and convenience. Based on these findings, we considered $\mathrm{T}_{3}$ as a more stable and reliable factor, and used $\mathrm{T}_{3}$ rather than $\mathrm{fT}_{3}$ for the analysis. Third, more than half of our study population, mostly having CV complications, were taking beta blockers. Patients with renal failure, especially those on dialysis treatment, have a considerably high prevalence of $\mathrm{CV}$ complications due to uremic toxin, volume overload, chronic inflammation, oxidative stress, and abnormal bone metabolism (34). In fact, 87\% of the patients had hypertension and $36.5 \%$ of the patients had underlying CV complications in this study. Even though beta blockers are known to affect serum $\mathrm{T}_{3}$ concentrations, we inferred that excluding these patients would result in selection bias. Moreover, the proportion of patients on beta blockers was not different between the 'lower' and 'higher' $\mathrm{T}_{3}$ groups, and beta blocker usage was not significantly associated with serum $\mathrm{T}_{3}$ concentrations in the correlation analysis $(r=0.062, P=0.180)$. Furthermore, in the linear regression analysis, beta blocker usage was not a significant determinant of serum $\mathrm{T}_{3}$ concentrations $(R=0.043, P=0.093)$. Fourth, there was a disparity in the timing of laboratory measurements and echocardiography. As has been mentioned above, since there was an extremely wide variation in the biochemical data at 0 month due to diverse patients' conditions to start $\mathrm{HD}$, we used the laboratory data collected at 3 months after HD commencement, when correction of uremia, anemia, and acidosis was considered to be sufficiently achieved. On the other hand, based on the results of previous studies demonstrating that LVMI, LVEF, and fractional shortening are improved after long-term $\mathrm{HD}$ of 12-36 months $(35,36)$, correction of malnutrition, anemia, acidosis, and thyroid hormone might influence the cardiac function at 3 months. However, no studies have yet shown significant changes in echocardiographic findings within 3 months of short-term dialysis. In this study, echocardiography was carried out not at the start of dialysis but close to the time of discharge, when patients were considered to be in euvolemic state. In fact, the mean interval between dialysis commencement and echocardiography was 19.7 days in our patients. In addition, since most incident HD patients had to undergo maintenance $\mathrm{HD}$ at private clinics in Korea and to pay an additional fee of \$300-350 for echocardiography, it was not easy to perform echocardiography at outpatient clinics after 3 months of initial dialysis. Therefore, we inevitably used 3-month laboratory data and baseline (about 3 weeks after the start of HD) echocardiographic parameters. Fifth, since only 3-month laboratory data were included in the analysis, it was difficult to determine the impact of the change in $\mathrm{T}_{3}$ levels on patients' clinical outcomes. Meuwese et al. (32) assessed the association between baseline and trimestral variation in $\mathrm{T}_{3}$ levels and mortality in 210 prevalent HD patients and found that not only basal low $\mathrm{T}_{3}$ but also persistently low $\mathrm{T}_{3}$ was significantly associated with increased mortality risk, especially due to $\mathrm{CV}$ causes. While the HRs for all-cause and CV mortality were higher in patients with persistently low $\mathrm{T}_{3}$ than in patients with low basal $\mathrm{T}_{3}$, there was still a significant association of low basal $\mathrm{T}_{3}$ with mortality. Sixth, all-cause and especially CV mortality rates in this study were relatively low. A small number of events may limit the power of statistical analyses in identifying the independent predictors of all-cause and especially $\mathrm{CV}$ mortality. Finally, the follow-up duration was relatively short and thus a sufficient number of patients could not be recruited for a longitudinal study. Since this prospective observation cohort study is still ongoing, the long-term association between $\mathrm{T}_{3}$ levels and mortality can be elucidated in the future. Despite these limitations, to our knowledge, this study is the first to investigate the association of $\mathrm{T}_{3}$ levels with echocardiographic findings and various nutritional parameters, including albumin levels, SGA score, LBM-Cr, and nPCR, and their impact on all-cause and CV mortality in a large, ethnically homogenous incident HD patient cohort.

In conclusion, low $\mathrm{T}_{3}$ is a significant independent risk factor for all-cause mortality in incident HD patients, and its impact on mortality may be attributed, in part, to malnutrition and cardiac dysfunction. 


\section{Supplementary data}

This is linked to the online version of the paper at http://dx.doi.org/ 10.1530/EJE-13-0540.

\section{Declaration of interest}

The authors declare that there is no conflict of interest that could be perceived as prejudicing the impartiality of the research reported.

\section{Funding}

This research did not receive any specific grant from any funding agency in the public, commercial, or not-for-profit sector.

\section{Acknowledgements}

The authors acknowledge the Korea Healthcare Technology R\&D Project, Ministry for Health, Welfare \& Family Affairs, Republic of Korea (A084001), for the support provided through a grant.

\section{References}

1 Iglesias P \& Diez JJ. Thyroid dysfunction and kidney disease. European Journal of Endocrinology $2009 \mathbf{1 6 0} 503-515$. (doi:10.1530/EJE-08-0837)

2 den Hollander JG, Wulkan RW, Mantel MJ \& Berghout A. Correlation between severity of thyroid dysfunction and renal function. Clinical Endocrinology 200562 423-427. (doi:10.1111/ j.1365-2265.2005.02236.x)

3 Shin DH, Lee MJ, Kim SJ, Oh HJ, Kim HR, Han JH, Koo HM, Doh FM, Park JT, Han SH et al. Preservation of renal function by thyroid hormone replacement therapy in chronic kidney disease patients with subclinical hypothyroidism. Journal of Clinical Endocrinology and Metabolism $2012 \quad 972732-2740$. (doi:10.1210/jc.2012-1663)

4 van Hoek I \& Daminet S. Interactions between thyroid and kidney function in pathological conditions of these organ systems: a review. General and Comparative Endocrinology $2009160205-215$. (doi:10.1016/j.ygcen.2008.12.008)

5 Song SH, Kwak IS, Lee DW, Kang YH, Seong EY \& Park JS. The prevalence of low triiodothyronine according to the stage of chronic kidney disease in subjects with a normal thyroidstimulating hormone. Nephrology, Dialysis, Transplantation 2009 24 1534-1538. (doi:10.1093/ndt/gfn682)

6 Ozen KP, Asci G, Gungor O, Carrero JJ, Kircelli F, Tatar E, Sevinc Ok E, Ozkahya M, Toz H, Cirit M et al. Nutritional state alters the association between free triiodothyronine levels and mortality in hemodialysis patients. American Journal of Nephrology 201133 305-312. (doi:10.1159/000324883)

7 Chopra IJ. Nonthyroidal illness syndrome or euthyroid sick syndrome? Endocrine Practice 19962 45-52. (doi:10.4158/ EP.2.1.45)

8 Wiederkehr MR, Kalogiros J \& Krapf R. Correction of metabolic acidosis improves thyroid and growth hormone axes in haemodialysis patients. Nephrology, Dialysis, Transplantation 200419 1190-1197. (doi:10.1093/ndt/gfh096)

9 Zoccali C, Tripepi G, Cutrupi S, Pizzini P \& Mallamaci F. Low triiodothyronine: a new facet of inflammation in end-stage renal disease. Journal of the American Society of Nephrology 200516 2789-2795. (doi:10.1681/ASN.2005040356)

10 Malyszko J, Malyszko JS, Pawlak K \& Mysliwiec M. Thyroid function, endothelium, and inflammation in hemodialyzed patients: possible relations? Journal of Renal Nutrition $2007 \mathbf{1 7}$ 30-37. (doi:10.1053/j.jrn.2006.07.003)

11 Yilmaz MI, Sonmez A, Karaman M, Ay SA, Saglam M, Yaman H, Kilic S, Eyileten T, Caglar K, Oguz Y et al. Low triiodothyronine alters flow-mediated vasodilatation in advanced nondiabetic kidney disease. American Journal of Nephrology 201133 25-32. (doi:10.1159/000322581)

12 Zoccali C, Benedetto F, Mallamaci F, Tripepi G, Cutrupi S, Pizzini P, Malatino LS, Bonanno G \& Seminara G. Low triiodothyronine and cardiomyopathy in patients with end-stage renal disease. Journal of Hypertension 200624 2039-2046. (doi:10.1097/01. hjh.0000244954.62362.8f)

13 Kozdag G, Ural D, Vural A, Agacdiken A, Kahraman G, Sahin T, Ural E \& Komsuoglu B. Relation between free triiodothyronine/free thyroxine ratio, echocardiographic parameters and mortality in dilated cardiomyopathy. European Journal of Heart Failure 20057 113-118. (doi:10.1016/j.ejheart.2004.04.016)

14 Pingitore A, Landi P, Taddei MC, Ripoli A, L’Abbate A \& Iervasi G. Triiodothyronine levels for risk stratification of patients with chronic heart failure. American Journal of Medicine 2005118 132-136. (doi:10.1016/j.amjmed.2004.07.052)

15 Scoscia E, Baglioni S, Eslami A, Iervasi G, Monti S \& Todisco T. Low triiodothyronine $\left(\mathrm{T}_{3}\right)$ state: a predictor of outcome in respiratory failure? Results of a clinical pilot study. European Journal of Endocrinology 2004151 557-560. (doi:10.1530/eje.0.1510557)

16 Malik R \& Hodgson $\mathrm{H}$. The relationship between the thyroid gland and the liver. QJM: Monthly Journal of the Association of Physicians 200295 559-569. (doi:10.1093/qjmed/95.9.559)

17 Zoccali C, Mallamaci F, Tripepi G, Cutrupi S \& Pizzini P. Low triiodothyronine and survival in end-stage renal disease. Kidney International 2006 70 523-528. (doi:10.1038/sj.ki.5001566)

18 Enia G, Panuccio V, Cutrupi S, Pizzini P, Tripepi G, Mallamaci F \& Zoccali C. Subclinical hypothyroidism is linked to micro-inflammation and predicts death in continuous ambulatory peritoneal dialysis. Nephrology, Dialysis, Transplantation 200722 538-544. (doi:10.1093/ndt/gfl605)

19 Rotondi M, Netti GS, Rosati A, Mazzinghi B, Magri F, Ronconi E, Becherucci F, Pradella F, Salvadori M, Serio M et al. Pretransplant serum $\mathrm{FT}_{3}$ levels in kidney graft recipients are useful for identifying patients with higher risk for graft failure. Clinical Endocrinology 200868 220-225. (doi:10.1111/j.1365-2265. 2007.03022.x)

20 Squire CR. Methods for the investigation of thyroid function. Methods in Molecular Biology 2006324 91-108. (doi:10.1385/ 1-59259-986-9-91)

21 Kilinc AS, Duzoylum A, Uncugil CF \& Yucel D. Falsely increased free triiodothyronine in sera stored in serum separator tubes. Clinical Chemistry 200248 2296-2297.

22 Stevenson HP, Archbold GP, Johnston P, Young IS \& Sheridan B. Misleading serum free thyroxine results during low molecular weight heparin treatment. Clinical Chemistry $1998 \mathbf{4 4} 1002-1007$.

23 Michels WM, Grootendorst DC, Verduijn M, Elliott EG, Dekker FW \& Krediet RT. Performance of the Cockcroft-Gault, MDRD, and new CKD-EPI formulas in relation to GFR, age, and body size. Clinical Journal of the American Society of Nephrology 2010 5 1003-1009. (doi:10.2215/CJN.06870909)

24 Steiber AL, Kalantar-Zadeh K, Secker D, McCarthy M, Sehgal A \& McCann L. Subjective Global Assessment in chronic kidney disease: a review. Journal of Renal Nutrition 200414 191-200.

25 Noori N, Kovesdy CP, Bross R, Lee M, Oreopoulos A, Benner D, Mehrotra R, Kopple JD \& Kalantar-Zadeh K. Novel equations to estimate lean body mass in maintenance hemodialysis patients. American Journal of Kidney Diseases 201157 130-139. (doi:10.1053/j.ajkd.2010.10.003)

26 Jindal KK \& Goldstein MB. Urea kinetic modelling in chronic hemodialysis: benefits, problems, and practical solutions. Seminars in Dialysis 19881 82-85. (doi:10.1111/j.1525-139X.1988.tb00710.x)

27 Devereux RB, Alonso DR, Lutas EM, Gottlieb GJ, Campo E, Sachs I \& Reichek N. Echocardiographic assessment of left ventricular hypertrophy: comparison to necropsy findings. American Journal of Cardiology 198657 450-458. (doi:10.1016/0002-9149(86) 90771-X)

28 Klein I \& Ojamaa K. Thyroid hormone and the cardiovascular system. New England Journal of Medicine 2001344 501-509. (doi:10.1056/NEJM200105103441901) 
29 Klein I \& Danzi S. Thyroid disease and the heart. Circulation 2007 116 1725-1735. (doi:10.1161/CIRCULATIONAHA.106.678326)

30 Tatar E, Kircelli F, Asci G, Carrero JJ, Gungor O, Demirci MS, Ozbek SS, Ceylan N, Ozkahya M, Toz H et al. Associations of triiodothyronine levels with carotid atherosclerosis and arterial stiffness in hemodialysis patients. Clinical Journal of the American Society of Nephrology 20116 2240-2246. (doi:10.2215/ CJN.02540311)

31 Carrero JJ, Qureshi AR, Axelsson J, Yilmaz MI, Rehnmark S, Witt MR, Barany P, Heimburger O, Suliman ME, Alvestrand A et al. Clinical and biochemical implications of low thyroid hormone levels (total and free forms) in euthyroid patients with chronic kidney disease. Journal of Internal Medicine 2007262 690-701. (doi:10.1111/j.1365-2796.2007.01865.x)

32 Meuwese CL, Dekker FW, Lindholm B, Qureshi AR, Heimburger O, Barany P, Stenvinkel P \& Carrero JJ. Baseline levels and trimestral variation of triiodothyronine and thyroxine and their association with mortality in maintenance hemodialysis patients. Clinical Journal of the American Society of Nephrology 20127 131-138. (doi:10.2215/CJN.05250511)
33 Jaume JC, Mendel CM, Frost PH, Greenspan FS \& Laughton CW. Extremely low doses of heparin release lipase activity into the plasma and can thereby cause artifactual elevations in the serumfree thyroxine concentration as measured by equilibrium dialysis. Thyroid 19966 79-83. (doi:10.1089/thy.1996.6.79)

34 Schiffrin EL, Lipman ML \& Mann JF. Chronic kidney disease: effects on the cardiovascular system. Circulation $2007 \mathbf{1 1 6} 85-97$. (doi:10.1161/CIRCULATIONAHA.106.678342)

35 Covic A, Mardare NG, Ardeleanu S, Prisada O, Gusbeth-Tatomir P \& Goldsmith DJ. Serial echocardiographic changes in patients on hemodialysis: an evaluation of guideline implementation. Journal of Nephrology 200619 783-793.

36 Foley RN, Parfrey PS, Kent GM, Harnett JD, Murray DC \& Barre PE. Serial change in echocardiographic parameters and cardiac failure in end-stage renal disease. Journal of the American Society of Nephrology $200011912-916$.

Received 10 May 2013

Accepted 12 July 2013 